\title{
Reservation and Status Sensing Multiple Access Protocol in Slotted CDMA Systems
}

\author{
Intaek Lim, Youngtae Ryu, Member, KIMICS
}

\begin{abstract}
This paper proposes a medium access control protocol for integrated voice and data services in slotted CDMA systems. The proposed protocol, which is named as RCSSMA (Reservation Code and Status Sensing Multiple Access), adopts a code reservation and status sensing schemes. RCSSMA protocol gives higher access priority to the voice traffic than data traffic for reducing the packet dropping probability. The voice terminal reserves an available spreading code to transmit voice packets during a talkspurt, whereas the data terminal transmits a packet over one of the available spreading codes that are not reserved by the voice terminals. In this protocol, the voice packets never contend with the data packets. Packet dropping probability and average data packet transmission delay are analyzed using a Markov chain model.
\end{abstract}

Index Terms-MAC protocol, Integrated service, Slotted CDMA, SSMA

\section{INTRODUCTION}

In wireless communication networks, many researches were carried out on code division multiple access (CDMA). CDMA systems offer the potential of high spectrum efficiency, soft capacity, multipath-resistance, and inherent frequency diversity [1][2][3]. Among many issues related to wireless communication network, medium access control (MAC) protocol that coordinates the multiple access of shared medium is one of the most important issues. MAC protocol might be enough efficient and robust to support the integrated voice and data service and to meet the quality of service requirements for both services. Therefore, the purpose of this paper is to propose an MAC protocol for integrated voice and data local wireless CDMA systems, called reservation code and status sensing multiple access (RCSSMA).

For integrated voice and data services, packet-switched network architecture seems to be the general choice. In conventional packet radio network based on TDMA system, there are several types of MAC protocols such as packet reservation multiple access (PRMA), statussensing multiple access ( $\mathrm{S}^{2} \mathrm{MA}$ ), and etc [4][5][6]. Basically, voice packets are transmitted periodically by

\footnotetext{
Manuscript received July 27, 2010; revised August 30, 2010; accepted September 6, 2010.

Intaek Lim is with the Department of Embedded IT, Pusan University of Foreign Studies, Busan, 608-738, Korea (Email: itlim@pufs.ac.)

Youngtae Ryu is with the Department of e-Business, Pusan University of Foreign Studies, Busan, 608-738, Korea (Email: youngtae@pufs.ac.)
}

reserving a slot in frame, whereas data packets are transmitted with slotted ALOHA scheme.

In [8], Tan et al. proposed reservation-code multiple access (RCMA) protocol, which apply a reservation random-access (RRA) protocol to spread spectrum multiple access systems. RRA protocols are special cases of random access protocols and have been developed for TDMA packet radio networks [6]. RCMA protocol can simplify code tracking hardware of the base station by allowing all terminals to share a group of spreading codes on a contention basis. Voice terminals, however, have to contend with data terminals for reserving a spreading code. This is a main cause of increasing voice dropping, especially when the voice traffic is dominant. Soroushnejad et al. proposed multiple access strategy for voice and data integrated CDMA packet radio network [7]. In this protocol, mobile terminals have to be assigned a unique spreading code at the system deployment stage. This transmitter-oriented code assignment has an advantage of no packet collision. However, the required number of spreading codes increases with the growing population of users, so code tracking and synchronization systems of the base station become complex.

The RCSSMA protocol results from applying a reservation random-access (RRA) and a status-sensing multiple access ( $\mathrm{S}^{2} \mathrm{MA}$ ) to slotted CDMA systems with a small coverage area. This protocol assumes that the uplink channels are composed of the time slots and spreading codes based on the wideband time-division and codedivision multiple accesses [9]. The time axis of uplink channel is divided into slots that are grouped into frames; a time slot has a group of spreading codes shared by all users.

This paper is organized as follows. The detailed descriptions of proposed protocol are described in Section II. In Section III, we analyze the performances of proposed protocol with a Markov chain mode. The numerical results are investigated in Section IV, and finally Section V concludes this paper.

\section{RCSSMA PROTOCOL}

\section{A. Slot and frame structure of uplink}

Fig.1 shows an example of the uplink frame format. A frame consists of $k_{0}$ time slots. For each slot, there are $m$ shared spreading codes $\left\{C_{i}, i=1, \ldots, m\right\}$ that are orthogonal to each other. Therefore, the total number of available channels in an uplink frame is $m^{*} k_{0}$. 
Voice and data terminals share a group of spreading codes to transmit a packet to the base station. Each code can be identified as "reserved" or "available" based on the feedback information from the base station at the end of each slot. A reserved code is exclusively assigned to the voice terminal until a talkspurt ends. An available code can be used by either voice terminals that want to reserve a code or data terminals that have data packets to transmit.

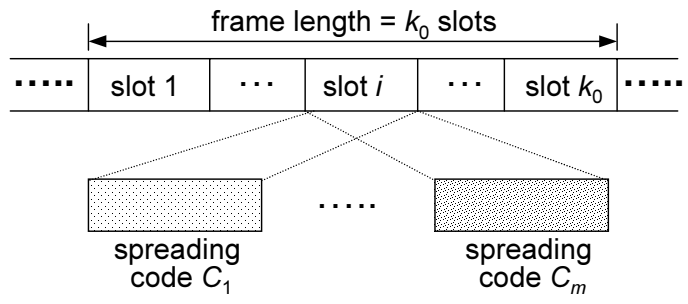

Fig.1. Uplink frame structure.

A packet generated by voice or data terminal can be transmitted during one slot with one spreading code. The uplink packet structure is illustrated in Fig.2. The user information will be encapsulated into a packet with additional overheads. The preamble is used for a spreading code acquisition by the base station. The datafield is composed of a TOP (Type of Packet), a header, and an information subfield. The TOP subfield is used to classify the packet type (i.e., voice, data, or reservationrequest packet). The header subfield contains terminal identifier, packet sequence number, etc. The reservationrequest packet also contains voice information.

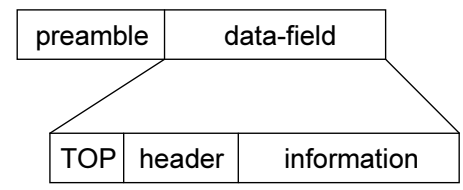

Fig.2. Packet format; TOP : Type of Packet.

\section{B. Voice subsystem operation}

Before describing the operation of voice subsystem, it is necessary to describe voice traffic model. For the voice terminal with a slow speech activity detector (SAD), its voice source can be characterized by a two-state model, as shown in Fig.3.

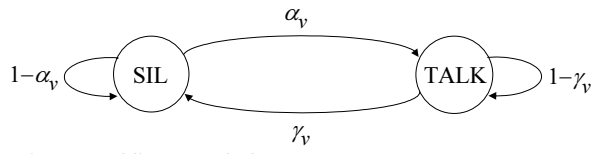

Fig.3. Voice traffic model.

For describing the voice traffic model, the following four parameters are required. These are the mean duration of a silence $t_{1}$, the mean duration of a talkspurt $t_{2}$, the transition probability from the silence state to the talking state $\alpha_{v}$, and the inverse transition probability $\gamma_{v}$. The talkspurt and silence is assumed to have exponentially distributed duration, which is statistically independent of one another.
Thus, the parameters $\alpha_{v}$ and $\gamma_{v}$ are represented as [6]

$$
\begin{aligned}
& \alpha_{v}=1-e^{-\tau / t_{1}} \\
& \gamma_{v}=1-e^{-\tau / t_{2}}
\end{aligned}
$$

where $\tau$ is the duration of a slot.

During a talkspurt, voice terminal generates voice packets with the period identical to the frame duration. Therefore, to transmit voice packet, the terminal needs to be guaranteed one channel (in this system, a spreading code in a slot) per frame.

When beginning a new talkspurt, the terminal enters into the reservation-contending state and tries to reserve a channel. The terminal in the reservation-contending state selects randomly an available spreading code in the next slot and transmits a reservation-request packet with the transmission permission probability $\beta$. If the base station captures the preamble field of the reservation-request packet, it immediately broadcasts an acknowledgement. The acknowledgement contains the spreading code information of current slot. If two or more voice terminals transmit the reservation-request packet with the same channel, a packet collision occurs. In this case, the base station cannot capture the preamble field and thus does not send an acknowledgement.

If the voice terminal receives an acknowledgement, it enters into the reservation state. The terminal in the reservation state transmits voice packets over the reserved channel of the subsequent frames until it ends a talkspurt. If the terminal decides not to transmit the reservation-request packet (with probability 1- $\beta$ ), or if it fails to reserve even in the case of transmission permission (for instance, by a collision or by a wireless channel error), it tries to reserve a spreading code in the next slot. If the voice terminal does not receive an acknowledgement, it does not transmit the remaining field of the reservation-request packet.

After a talkspurt completion, voice terminal does not access the reserved code. If the base station does not detect a packet from a reserved code (i.e., it fails the code acquisition of the reserved code), it regards the code as an available code, which can be used by any terminal in the next frame.

In the voice traffic, the transmission delay of voice packets causes a serious problem in the quality of service. If the terminal cannot transmit voice packets until any given speech delay constraint ( $W_{\max }$ slots), these packets must be dropped. In this paper, the packet dropping probability $\left(P_{\text {drop }}\right)$ is considered as the performance measure of voice traffic.

\section{Data subsystem operation}

In RCSSMA protocol, the operation of data subsystem is similar to the slotted CDMA_ALOHA scheme [10]. The data subsystem does not use the reservation scheme but the spreading code status-sensing scheme. When a data terminal in the idle state generates a packet, it then enters into the contending state. The data terminal in the 
contending state selects a spreading code in the current slot, which was not already reserved by a voice terminal in the reservation state and is not currently reserved by a voice terminal in the reservation-contending state. The base station broadcasts the code reserving status of current slot after correlating the preamble field. The transmission of data packet, therefore, delays until the preamble field of the reservation-request packet. After receiving the code status information of the current slot from the base station, a data terminal transmits a packet with the transmission permission probability $\beta$. If the base station successfully captures a data packet, it also broadcasts an acknowledgement for the packet.

If the terminal receives an acknowledgement and has packets in its buffer, it remains in the contending state and tries to transmit another packet in the next slot. The terminal that receives an acknowledgement and does not have any packet to transmit goes into the idle state. If the terminal fails to transmit a packet, it remains in the same state and tries to send the same packet in the next slot.

In data service, to guarantee the quality of service, packets must be delivered although they are delayed. Therefore, the average packet delay $\left(D_{d}\right)$ is considered as the performance measure of data traffic.

\section{PERFORMANCE ANALYSIS}

\section{A. Voice subsystem}

The voice terminal is independent on the data terminal because the former has high access priority than the latter. Let $N_{v}$ the number of voice terminals.

At a given slot $t$, a voice terminal can remain in one of the following state: the silence state, the reservationcontending state, and the reservation state. Therefore, we can define the voice subsystem state $\left\{X_{v}^{t}=\left(R_{v}{ }^{t}, C_{v}{ }^{t}\right)\right\}$ as the number of voice terminals in the reservation state $\left(R_{v}{ }^{\dagger}\right)$ and in the reservation-contending state $\left(C_{v}{ }^{t}\right)$. Let $Q_{j k, r l}^{v}$ be the state transition probability from the state $(j, k)$ in a slot to the state $(r, l)$ in the following slot. This probability, which can be derived from the conditional probabilities that $v_{i}$ terminals in the silence state begin a new talkspurt, $v_{r}$ terminals in the reservation-contending state transmit the reservation-request packet through one of $x$ available codes, and $s_{v}$ packets succeed to reserve a spreading code, is given by

$$
\begin{aligned}
Q_{j k, r l}^{v}= & \sum_{x=0}^{m} \sum_{v_{i}=0}^{N_{v}-j-k} \sum_{v_{r}=0}^{k} \\
& \left\{\begin{array}{l}
\Phi\left(x, j, k_{0}, m\right) b\left(N_{v}-j-k, v_{i}, \alpha_{v}\right) b\left(k, v_{r}, \beta\right) \\
b\left(j+s_{v}, r, 1-\gamma_{v}\right) S\left(s_{v} \mid v_{r}, x\right)
\end{array}\right\}
\end{aligned}
$$

where

$$
\begin{aligned}
& s_{v}=k+v_{i}-l, 0 \leq s_{v} \leq \min \left(v_{r}, x\right) \\
& 0 \leq\{j, r\} \leq \min \left(N_{v}, m k_{0}\right), 0 \leq k \leq N_{v}-j, 0 \leq l \leq N_{v}-r \\
& b(n, i, p)=\left(\begin{array}{c}
n \\
i
\end{array}\right) p^{i}(1-p)^{n-i}
\end{aligned}
$$

In equation (2), the term $S(s \mid n, x)$ is the conditional probability that $s$ packets out of $n$ are successfully transmitted over one of $x$ available codes. The probability $S(s \mid n, x)$ can be recursively defined as follows [10]

$$
\begin{aligned}
S(s \mid n, x) & =\sum_{j=0}^{n}\left(\begin{array}{l}
n \\
j
\end{array}\right)\left(\frac{1}{x}\right)^{j}\left(1-\frac{1}{x}\right)^{n-j} \\
& \left\{\zeta_{j} S(s-1 \mid n-j, x-1)+\left(1-\zeta_{j}\right) S(s \mid n-j, x-1)\right\}
\end{aligned}
$$

where $\zeta_{1}=1$, and $\zeta_{j}=0(j \neq 1)$. And its initial conditions are given by

$$
\begin{array}{ll}
S(0 \mid 0, x)=1, S(1 \mid 0, x)=0, & \text { for } x \geq 0 \\
S(0 \mid n, 0)=1, S(1 \mid n, 0)=0, & \text { for } n \geq 0 \\
S(0 \mid 1, x)=0, S(1 \mid 1, x)=1, & \text { for } x \geq 1 \\
S(1 \mid n, 1)=0, S(0 \mid n, 1)=1, & \text { for } n \geq 2 \\
S(s \mid n, x)=0, & \text { for } s>\min (n, x)
\end{array}
$$

The term $\Phi\left(x, j, k_{0}, m\right)$ in equation (2) is the probability that $x$ spreading codes are available in a slot under the condition of $j$ reserved voice terminals. If $j$ is the number of voice terminals in the reservation state at the beginning of a given slot, then the probability that the codes within that slot are reserved by the voice terminals is determined by the following. Let $D\left(j, k_{0}, m\right)$ be the number of ways of distributing $j$ indistinguishable voice terminals into $k_{0}$ distinguishable slots under the restriction of $m$-code occupancy per slot (i.e., at any given slot, at most $m$ terminals can transmit packet). According to [11], $D\left(j, k_{0}, m\right)$ and $\Phi\left(x, j, k_{0}, m\right)$ are given by

$$
\begin{gathered}
D\left(j, k_{0}, m\right)=\sum_{i=0}^{k_{0}}(-1)^{i}\left(\begin{array}{c}
k_{0} \\
i
\end{array}\right)\left(\begin{array}{c}
j+k_{0}-i(m+1)-1 \\
k_{0}-1
\end{array}\right) \\
\Phi\left(x, j, k_{0}, m\right)=\frac{D\left(j-m+x, k_{0}-1, m\right)}{D\left(j, k_{0}, m\right)}
\end{gathered}
$$

where $j \leq m k_{0}, 0 \leq x \leq m, k_{0} \geq 1$.

Once the transition probability $Q_{j k, r l}^{v}$ is obtained, the steady state probability $\Pi_{r l}^{v}$ that $r$ voice terminals are in the reservation state and $l$ voice terminals are in the reservation-contending state is derived as [12]

$$
\begin{aligned}
& \Pi_{r l}^{v}=\sum_{j=0}^{\min \left(N_{v}, m k_{0}\right)} \sum_{k=0}^{N_{v}-j} Q_{j k, r l}^{v} \times \Pi_{j k}^{v} \\
& \min \left(\sum_{r=0}^{\left.N_{v}, m k_{0}\right)} \sum_{l=0}^{N_{v}-r} \Pi_{r l}^{v}=1\right.
\end{aligned}
$$

The performance measure of voice subsystem in RCSSMA protocol is the voice packet dropping probability. The descriptive mechanisms for the packet dropping are as follows. Let $L$ be the number of packets generated during a talkspurt. In a talkspurt, no packets are dropped if the voice terminal reserves before $W_{\max }$ slots. If it fails in reservation during $W_{\max }$ slots, the terminal drops the first packet. The second packet is generated exactly $k_{0}$ slots after the first packet. Therefore, the terminal drops the second packet if it is still in the reservation-contending state after $W_{\max }+k_{0}$ slots. If the terminal can not reserve after $W_{\max }+(L-1) k_{0}$ slots, it drops the entire packets of a talkspurt.

It is assumed that voice terminal generates $L$ packets 
during a talkspurt. The probability that $k$ packets are dropped in a talkspurt is

$$
\begin{aligned}
& \operatorname{Pr}\left\{n_{\text {drop }}=k \mid L\right\} \\
& \quad= \begin{cases}1-f^{W_{\max }} & , \text { for } k=0 \\
f^{W_{\max }+(k-1) k_{0}}-f^{W_{\max }+k k_{0}} & , \text { for } 1 \leq k \leq L-1 \\
f^{W_{\max }+(L-1) k_{0}} & , \text { for } k=L\end{cases}
\end{aligned}
$$

where $f$ is the probability that a reservation-contending terminal fails to reserve a code and defined as

$$
f=1-\sum_{r=0}^{\min \left(N_{v}, m k_{0}\right)} \sum_{l=0}^{N_{v}-r} \sum_{v_{r}=0}^{l} \sum_{x=1}^{m}\left\{\begin{array}{l}
\Phi\left(x, r, k_{0}, m\right) b\left(l, v_{r}, \beta\right) \\
S\left(v_{r} \mid v_{r}, x\right) \Pi_{r l}^{v}
\end{array}\right\}
$$

By averaging the equation (7) over $L$, the mean number of dropped packets in a talkspurt is given by

$$
\begin{aligned}
E\left\{n_{\text {drop }}\right\} & =\sum_{L=1}^{\infty} \sum_{k=0}^{L} k \cdot \operatorname{Pr}\left\{n_{\text {drop }} \mid L\right\} \cdot \operatorname{Pr}\{L\} \\
& =\frac{f^{W_{\max }}}{1-\left(1-\gamma_{f}\right) f^{k_{0}}}
\end{aligned}
$$

where $\operatorname{Pr}\{L\}$ is the probability that there are $L$ packets in a talkspurt. The probability $\operatorname{Pr}\{L\}$ is defined as

$$
\operatorname{Pr}\{L\}=\gamma_{f}\left(1-\gamma_{f}\right)^{L-1}
$$

where $\mathrm{L} \geq 1$. In equation (10), $\gamma_{f}$ is the probability that a talkspurt ends in a frame and is represented

$$
\gamma_{f}=1-\left(1-\gamma_{v}\right)^{k_{0}}
$$

where $\gamma_{v}$ is given in equation (1).

It is defined that the packet dropping probability is the ratio of dropped packets to the total number of generated voice packets. Then the packet dropping probability is

$$
P_{\text {drop }}=\gamma_{f} \cdot \frac{f^{W_{\max }}}{1-\left(1-\gamma_{f}\right) f^{k_{0}}}
$$

\section{B. Data subsystem}

The data terminal attempts to transmit a packet with an available code that is not used by a voice terminal in the reservation-contending state or the reservation state. The state of a data terminal, therefore, is dependent on the state of the voice terminal. In this paper, the data buffer size is assumed to be sufficiently large enough to ignore the data packet blocking caused by the lack of buffer.

Let $N_{d}$ be the number of data terminals in the system. And let the data subsystem state be $\left\{X_{d}^{t}=\left(C_{d}^{t}, R_{v}^{t}, C_{v}{ }^{t}\right)\right\}$ where $C_{d}^{t}$ is the number of the contending data terminals. The state transition probability $Q^{d}{ }_{i j k, b r l}$ that the system state changes from $(i, j, k)$ in a slot to $(b, r, l)$ in the following slot is given by

$$
\begin{aligned}
& Q_{i j k, b r l}^{d}=\sum_{x=0}^{m} \sum_{v_{i}=0}^{N_{v}-j-k} \sum_{v_{r}=0}^{k} \sum_{d_{i}=0}^{N_{d}-i} \sum_{d_{b}=0}^{i} \\
&\left\{\begin{array}{l}
\Phi\left(x, j, k_{0}, m\right) b\left(N_{v}-j-k, v_{i}, \alpha_{v}\right) b\left(k, v_{r}, \beta\right) \\
b\left(j+s_{v}, r, 1-\gamma_{v}\right) b\left(N_{d}-i, d_{i}, \alpha_{d}\right) b\left(i, d_{b}, \beta\right) \\
S\left(s_{v} \mid v_{r}, x\right) S\left(s_{d} \mid d_{b}, x-s_{v}\right)
\end{array}\right\}
\end{aligned}
$$

where

$$
\begin{aligned}
& s_{v}=k+v_{i}-l, s_{d}=i+d_{i}-b \\
& 0 \leq s_{v} \leq \min \left(v_{r}, x\right), 0 \leq s_{d} \leq \min \left(d_{b}, x-s_{v}\right) \\
& 0 \leq\{i, b\} \leq N_{d} \\
& 0 \leq\{j, r\} \leq \min \left(N_{v}, m k_{0}\right), 0 \leq k \leq N_{v}-j, 0 \leq l \leq N_{v}-r
\end{aligned}
$$

In equation (13), $\alpha_{d}$ is the data packet arrival rate in a slot. Once the transition probability of data subsystem is obtained, the steady state probability can be derived as

$$
\begin{aligned}
& \Pi_{b r l}^{d}=\sum_{i=0}^{N_{d}} \sum_{j=0}^{\min \left(N_{v}, m k_{0}\right)} \sum_{k=0}^{N_{v}-j} Q_{i j k, b r l}^{d} \times \Pi_{i j k}^{d} \\
& \sum_{b=0}^{N_{d}} \min \left(\sum_{r=0}^{N_{v}, m k_{0}} \sum_{l=0}^{N_{v}-r} \Pi_{b r l}^{d}=1\right.
\end{aligned}
$$

The performance measures of data traffic are the system throughput and the average packet delay. Let the system throughput be the total number of data packets successfully transmitted in a slot. Then the system throughput can be defined as follows

$$
\eta_{d}=\alpha_{d}\left(N_{d}-\sum_{b=0}^{N_{d}} \sum_{r=0}^{\min \left(N_{v}, m k_{0}\right)} \sum_{l=0}^{N_{v}-r} b \Pi_{b r l}^{d}\right)
$$

Let the average packet delay be the elapsed time of a data packet from the generation to the successful transmission. Then the average packet delay can be defined as

$$
D_{d}=\frac{N_{d}}{\eta_{d}}-\frac{1}{\alpha_{d}}+1
$$

\section{ANALYSIS RESULTS}

The nominal values of parameters in the numerical analysis and simulation are listed in Table 1. In RCSSMA protocol, frame duration is identical to the packet generation period of voice terminals. With the values of parameters listed in Table 1, slot duration is $5 \mathrm{msec}$, and thus a frame consists of 5 slots. A voice packet, which is failed to reserve a channel during 10 slots, will be dropped.

TABLE 1.

PARAMETERS FOR PERFORMANCE ANALYSIS.

\begin{tabular}{l|l}
\hline Parameter & Value \\
\hline CDMA channel rate & $3.84 \mathrm{Mcps}$ \\
Uplink channel bit rate & $192 \mathrm{Kbps}$ \\
Frame duration & $20 \mathrm{msec}$ \\
Speech delay constraint & $40 \mathrm{msec}$ \\
Voice sampling rate & $32 \mathrm{Kbps}$ \\
Packet overhead & $64 \mathrm{bits}$ \\
Mean silence duration $\left(t_{1}\right)$ & $1.35 \mathrm{sec}$ \\
Mean talkspurt duration $\left(t_{2}\right)$ & $1.00 \mathrm{sec}$ \\
Number of spreading codes $(m)$ & Variable \\
Transmission permission probability $(\beta)$ & Variable \\
\hline
\end{tabular}


Fig.4 and Fig.5 show the voice packet dropping probabilities according to the number of voice terminals when $m=3$. These figures plotted the results compared with RCMA protocol. Collision of the reservation-request packet occurs more frequently in proportion to $N_{v}$, and therefore the voice packet dropping also increases. Assuming $P_{\text {drop }} \leq 0.01$, when the transmission permission probability $\beta$ is 0.1 , RCMA protocol can accommodate only 22 voice terminals because the reservation-request packet has to contend with data packets. But in the RCSSMA protocol, the reservation-request packets never contend with data packets. $P_{\text {drop }}$ in the proposed protocol, therefore, can accommodate about 43 voice terminals. As increasing $\beta$ to $0.3, P_{\text {drop }}$ is increased rapidly. This is because the increased contention probability leads to excessive collisions for large $\beta$. But as shown in Fig.5, RCSSMA protocol gives more stable performance than RCMA in spite of large $\beta$.

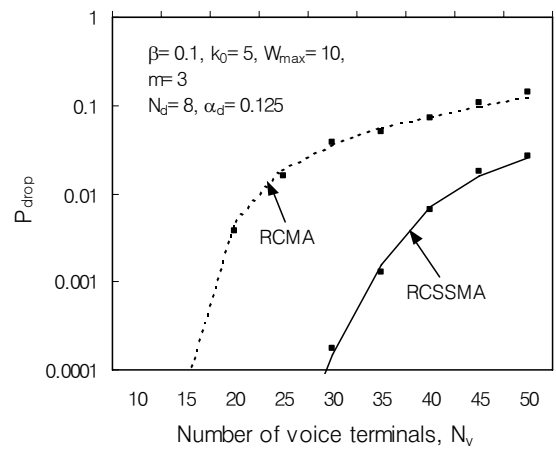

Fig.4. Packet dropping probability $P_{\text {drop }}$ vs. $N_{v}$.

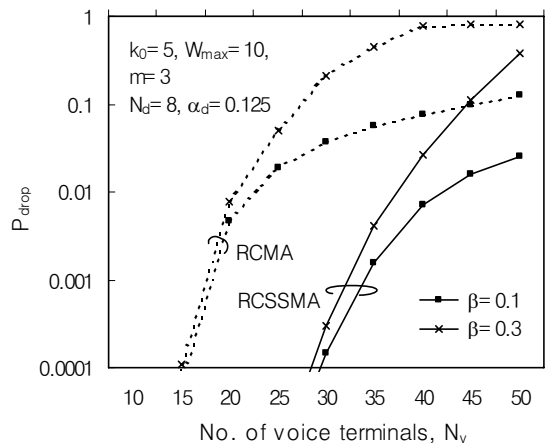

Fig.5. Effect of transmission permission probability on packet dropping probability according to $N_{v}$.

Fig.6 shows the effect of the transmission permission probability on the voice packet dropping probability when $m=2$ and $m=3$, respectively. The increase of $\beta$ up to a threshold value leads to the frequent reservation failure, and therefore $P_{\text {drop }}$ increases rapidly. From Fig.6, the threshold values of $\beta$ are 0.4 and 0.8 for the number of spreading codes $m=2$ and 3, respectively.

Fig.7 shows the relation between the total number of channels and the $P_{\text {drop }}$. The more the number of spreading codes is, the more the total number of channels is. Also as the number of slot increases, so the total number of channels does. Therefore $P_{\text {drop }}$ decreases for the large number of spreading codes and slots. By reducing the voice-coding rate, the number of slots in the uplink frame can be increased.

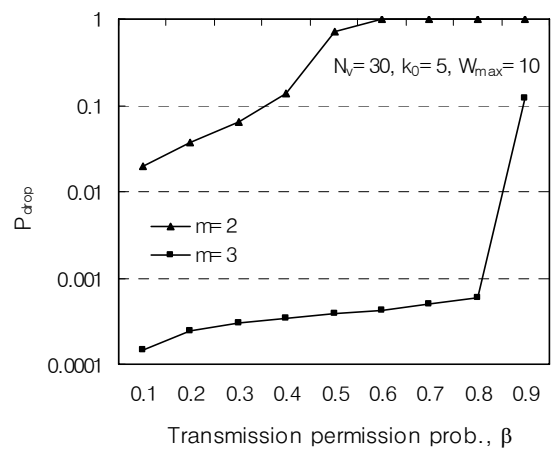

Fig.6. $P_{\text {drop }}$ vs. $\beta$ according to $m$.

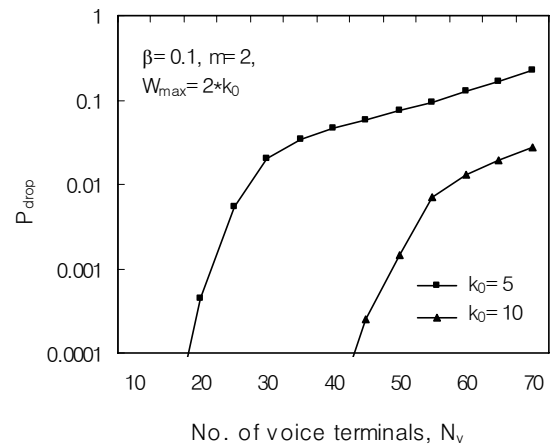

Fig.7. Packet dropping probability $P_{\text {drop }}$ vs. $N_{v}$ according to number of slots in the uplink frame $k_{0}$.

Fig. 8 shows the average data packet delay according to the number of spreading codes when $\beta=0.1$. If the number of spreading codes is small, almost all of the codes are reserved by the voice terminals because of its low access priority. Therefore, it leads the data packets to the excessive collision. However, as shown in these figures, the average delay is significantly improved by increasing the number of spreading codes.

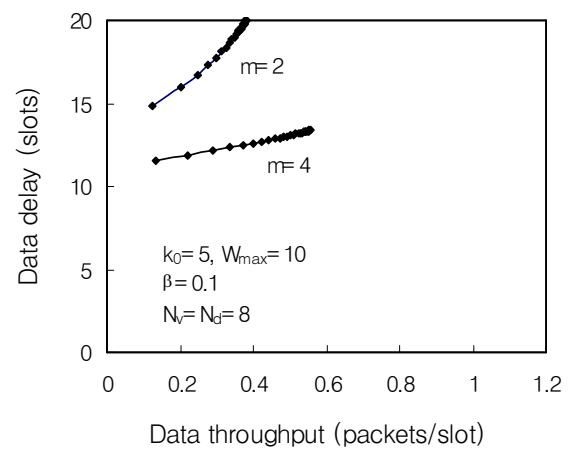

Fig.8. Average packet delay vs. $m$. 
Fig.9 illustrates the effects of the transmission permission probability on the average packet delay. As shown in Fig.9, when $m$ is small (i.e., $m=2$ ), the increase of $\beta$ up to a certain threshold value (approximately 0.3 ) causes the average delay to decrease continuously. However, the average data packet delay increases rapidly by increasing the transmission permission probability above the threshold. This is because at a given packet generation rate, the collision of data packets occurs frequently as data terminals try to transmit a packet with high probability. As shown in Fig.9, these effects do not occur when the number of spreading codes is large.

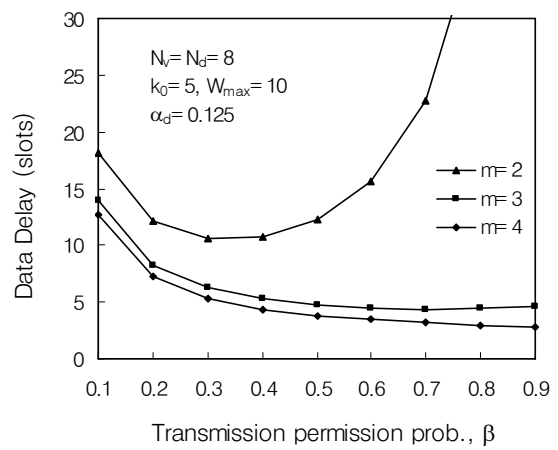

Fig.9. Effect of transmission permission probability on the average packet delay according to $m$.

\section{CONCLUSIONS}

In this paper, RCSSMA protocol has been proposed as a medium access control protocol for slotted CDMA systems supporting integrated voice and data services. The RCSSMA protocol allows all voice and data terminals to share a group of spreading codes on a reservation and a contention basis. This protocol accommodates the advantages of the reservation scheme for the voice traffic, the simplicity of random access scheme for the data traffic, and the multiple access capability of CDMA.

Markov chain analysis is used to evaluate the system behavior, leading to expressions for the voice packet dropping probability and the average data packet delay. The numerical analysis results have shown that the voice packet dropping probability and the average data packet delay are strongly dependent on the number of spreading codes. Increasing the number of spreading codes helps to raise the voice system capacity and improve the average data packet delay.

\section{REFERENCES}

[1] H. Jiang, W. Zhuang, X. Shen, and Q. Bi, "Quality-of-service provisioning and efficient resource utilization in CDMA cellular communications," IEEE J. on Selected Areas in Commun., vol. 24, no. 1, pp. 4-15, Jan. 2006.
[2] A. Baier, et al., "Design study for a CDMA-based third generation mobile radio systems," IEEE Jr. of Selected Areas in Commun., vol.12, no.4, pp.733-742, May 1994.

[3] M. B. Pursley, "The role of spread spectrum in packet radio networks," Proc. of IEEE, vol.75, no.1, pp.116-134, 1987.

[4] D. J. Goodman, et al., "Packet reservation multiple access for local wireless communications," IEEE Trans. on Commun., vol.37, no.8, pp.885-890, Aug. 1989.

[5] R. Al-Naami, and D. Saha, "Status sense multiple-access ( $\left.{ }^{2} \mathrm{MA}\right)$ communication," IEEE Trans. on Commun., vol.40, no.6, pp.9961002, Jun. 1996.

[6] S. Jangi, and L. F. Merakos, "Performance analysis of reservation and random access protocol for wireless access networks," IEEE Trans. on Commun., vol.42, no.2/3/4, pp.1223-1234, Feb./Mar./Apr. 1994.

[7] M. Soroushnejad, and E. Geraniotis, "Multiple-access strategies for an integrated voice/data CDMA packet radio networks," IEEE Trans. on Commun., vol.42, no.1/2/3, pp.934-945, Feb./Mar./Apr. 1995.

[8] L. Tan, and Q. T. Zhang, "A reservation random access protocol for voice/data integrated spread-spectrum multiple access systems," IEEE Jr. of Selected Areas on Commun., vol.14, no.9, pp.1717-1727, Dec. 1996.

[9] J. Perez-Romero, O. Sallent, and R. Agusti, "On the optimum traffic allocation in heterogeneous CDMA/TDMA networks," IEEE Trans. Wireless Commun., vol. 6, no. 9, pp. 3170-3174, Sept. 2007.

[10] I. Lim, "An MAC Protocol with Reservation and Channel Sensing for the Integrated Voice/Data Packet CDMA Networks", in Proc. of APCC/OECC99, pp.813-816, Oct. 1999, China.

[11] W. Feller, An introduction to probability theory and its applications, Vol.1, John Wiley \& Sons, pp.98-111, 1968.

[12] L. Kleinlock, Queueing Systems Volume 1: Theory, John Wiley \& Sons, pp.26-44, 1975.

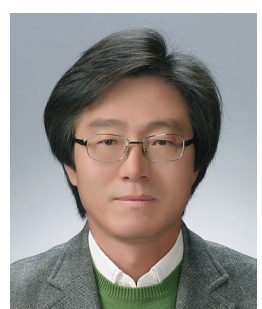

Intaek Lim received the B.S. degree in computer science from University of Ulsan, Ulsan, Korea, in 1984, and the M.S. degree in computer science and statistics from Seoul National University, Seoul, Korea, in 1986 . He received the $\mathrm{Ph}$. D. degree in computer engineering from University of Ulsan, Ulsan, Korea, in 1998. From 1986 to 1993, he was a Senior Researcher at Samsung Electronics Co., Ltd. In 1998, he joined the faculty at Pusan University of Foreign Studies, Busan, Korea, where he is currently a Professor in the Department of Embedded IT. From July 2006 to June 2007, He was a visiting scholar at Cleveland State University in USA. His research interests include the MAC protocol design, ad-hoc network, RFID, and mobile computing.

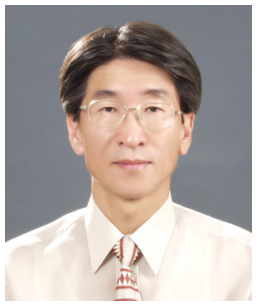

Youngtae Ryu is associate professor, Department of e-Business at the Pusan University of Foreign Studies. He received Ph.D.(Major in ATTD and Minor in BCIS) from the University of North Texas in 1997, MBA(Major in Management Science) from the Pusan National University in 1983 and MBA(Major in BCIS) from the University of North Texas in 1992, and Bachelor degree in Business Administration from the Pusan National University in 1980. His current research interests focus on Information Systems Research, Database Management System, System Analysis and Design, ERP, and Knowledge Management. 